\title{
GESTÃO DO CONHECIMENTO EM TEMPOS DE CRISE
}

\author{
Tácito Almeida de Lucca, MBA ${ }^{\mathbf{1}}$; \\ Neri dos Santos, Dr. Ing. ${ }^{2}$;
}

\begin{abstract}
In the last few decades, a series of crises of the most different natures have left scars in history and brought to light the vulnerability in which the population, organizations and governments find themselves when it comes to facing crises. The development of prevention, preparedness and recovery measures are key factors in reducing the frequency and severity of crises. Knowledge management (KM) has been recognized as an important multidiscipline capable of supporting crisis management. The qualitative methodology consisted of a rigorous systematic review of theoretical and empirical literature. The aim of the study was to build a set of elements, actions and artifacts from the fields of KM, risk management, crisis management, resulting in a prescriptive theoretical framework of processes with important implications for KM professionals.
\end{abstract}

Keywords: knowledge management; risks; crisis management; organizational crisis; framework.

Resumo: Nas últimas décadas uma série de crises das mais diferentes naturezas deixaram cicatrizes na história e trouxeram a tona a vulnerabilidade em que se encontram a população, organizações e governos no que tange o enfreitamento de crises. O desenvolvimento de medidas de prevenção, preparação e recuperação são fatores-chave para diminuir a frequência e a gravidade das crises. A gestão do conhecimento (GC) tem sido reconhecida como uma importante multidisciplina capaz de apoiar o gerenciamento de crises. A metodologia qualitativa consistiu em uma rigorosa revisão sistemática de literatura teórica e empírica. $\mathrm{O}$ objetivo do estudo foi construir um conjunto de elementos, ações e artefatos dos campos da GC, gerenciamento de riscos, gerenciamento de crises, resultando em um framework teórico prescritivo de processos com implicações importantes para os profissionais da GC.

Palavras-chave: gestão do conhecimento; riscos; gerenciamento de crises; crise organizacional; framework;

\footnotetext{
${ }^{1}$ Mestrando do Programa de Pós-Graduação em Engenharia e Gestão do Conhecimento - Universidade Federal de Santa Catarina (UFSC) Florianópolis - Brasil. Correo electrónico: tacitodelucca@ gmail.com

${ }^{2}$ Professor Sênior do Programa de Pós-Graduação em Engenharia e Gestão do Conhecimento - Universidade Federal de Santa Catarina (UFSC) Florianópolis - Brasil. Correo electrónico: nerisantos@gmail.com
} 


\section{INTRODUÇÃO}

Nas últimas décadas foram observadas uma série de crises das mais diferentes naturezas (desastres naturais, terrorismo, política, econômica, cibernética, corrupção, falhas de produtos, saúde) que atingiram desde o cidadão comum até nações inteiras, onde muitas delas, infelizmente, deixaram grandes cicatrizes no tempo. Essas crises tem trazido a tona a vulnerabilidade em que se encontram a população, organizações e governos no que tange o enfreitamento de crises.

Crises podem ameaçar severamente a integridade de uma organização (Wang \& Belardo, 2009) e o desenvolvimento de medidas de prevenção, preparação e recuperação são fatores-chave para diminuir a frequência e a gravidade das crises. Tais fatores podem ser fortalecidos antecipadamente durante a fase pré-crise ou reabastecidos quando o choque tiver passado (Hernantes, Rich, Laugé, Labaka, \& Sarriegi, 2013). Obter conhecimento correto, no momento certo, no lugar certo e, se possível, pelo custo certo, é fundamental para o sucesso de gerenciar qualquer situação de crise (Ponis \& Koronis, 2012).

Neste sentido a gestão do conhecimento (GC) tem sido reconhecida como uma importante multidisciplina capaz de apoiar a gestão da complexidade em tempos de crise, tornando-se essencial nestes períodos incertos (Coombs, 1999; Wang \& Belardo, 2009; Kyobe, 2010; Blackman, Kennedy, \& Ritchie, 2011; Ponis \& Koronis, 2012; Cania \& Korsita, 2015; Lauras, Truptil, \& Bénaben, 2015). Entretanto, poucos estudos vinculam explicitamente a gestão do conhecimento e o gerenciamento de crises (Wang \& Belardo, 2009; Ponis \& Koronis, 2012; Paraskevas, Altinay, McLean, \& Cooper, 2013).

O artigo é dividido em quatro seções: revisão de literatura; metodologia; resultados e análises; e considerações finais. Na primeira seção são apresentados conceitos sob diferentes perspectivas da GC, do gerenciamento de crises e as práticas e desafios da gestão do conhecimento em tempos de crise. Na parte subsequente é retratada a metodologia empregada. Na penúltima seção são divulgados os resultados e manifestadas suas análises. Para concluir o artigo, contudo não o tema em pauta, são realizadas considerações finais e indicados possíveis desdobramentos da pesquisa.

O objetivo do estudo foi construir um conjunto teórico de elementos, ações e artefatos dos campos da GC, gerenciamento de riscos, gerenciamento de crises, resultando em um framework teórico prescritivo de processos que fornece orientações gerais sobre os procedimentos da GC com implicações importantes para os profissionais que atuam com a GC em organizações públicas e privadas na prevenção, combate e recuperação de crises. 


\section{REVISÃO DE LITERATURA}

\subsection{GESTÃO DO CONHECIMENTO}

Estamos na era da economia do conhecimento, em que o conhecimento não é apenas uma característica essencial das sociedades pós-industriais, mas também o recurso crítico (Evans, 2003; e Kyobe, 2010) e propulsor de desenvolvimento econômico e sucesso dos indivíduos, organizações e nações. Atualmente, as organizações consideram o conhecimento como uma alavanca estratégica para a vantagem competitiva e o sucesso dos negócios parece depender cada vez mais da capacidade de avaliar, gerenciar, desenvolver e adquirir novos conhecimentos (Davenport \& Prusak, 1998; Paraskevas et al., 2013).

Conforme definido por Davenport e Prusak (1998), o conhecimento é uma mistura fluida de experiência estruturada, valores, informações contextuais, percepção de especialistas e intuição fundamentada que fornece um ambiente e estrutura para avaliar e incorporar novas experiências e informações. Ele se origina e é aplicado na mente de quem conhece. Nas organizações, muitas vezes fica incorporado não apenas em documentos ou repositórios, mas também em rotinas, processos, práticas e processos organizacionais.

Polanyi (1966) argumenta que existem dois tipos de conhecimento: tácito e explícito. O conhecimento tácito é uma forma de conhecimento que é altamente pessoal e profundamente enraizada em experiências, idéias, valores, modelos mentais e emoções individuais. Por outro lado, o conhecimento explícito ou conhecimento codificado é o conhecimento que se manifesta através da linguagem, símbolos, objetos e artefatos e que é formalizado na forma de relatórios, equações e especificações, sendo este mais facilmente transmitido entre indivíduos e equipes.

Esse conhecimento retido é parte integrante do processo do aprendizado organizacional. A retenção de conhecimento é um elemento central do conceito de memória organizacional, permitindo que as empresas incorporem conhecimento dentro da organização (Argote, McEvily, \& Reagans, 2003). A diferença entre o conhecimento que uma organização precisa e o conhecimento que ela possui é chamada de gap de conhecimento (Wang \& Belardo, 2009).

Zack (1999) divide o conhecimento organizacional em três categorias: central, avançado e inovador. O conhecimento central é o conhecimento básico que permite que uma empresa sobreviva no mercado a curto prazo. $\mathrm{O}$ conhecimento avançado fornece à empresa um conhecimento semelhante ao de seus rivais. O conhecimento inovador confere à empresa sua posição competitiva sobre seus rivais. Evans (2003) acrescenta que os ativos de conhecimento aumentam com o uso, enquanto os recursos materiais diminuem quando usados. 
A gestão do conhecimento é relativamente jovem e envolve a integração de várias disciplinas, incluindo ciência da computação e administração, sociologia, gestão de recursos humanos e estratégia (Blackman et al., 2011) e, portanto, se constitui em uma multidisciplina. A GC pode ser definida como um conjunto de processos sistêmicos para gerenciar o conhecimento das organizações a fim de criar, armazenar, disseminar e utilizar conhecimentos necessários para as atividades nos diferentes níveis organizacionais criando valor comercial e gerando vantagem competitiva (Davenport \& Prusak, 1998; Cania \& Korsita, 2015). Para a Norma ISO 30.401:2018 GC é uma abordagem composta pela criação, compartilhamento e aplicação de conhecimento com foco na ampliação de produtividade, do rendimento e do avanço organizacional. Para Bhatt (2001) a interação entre tecnologias, técnicas e pessoas permite que uma organização gerencie seu conhecimento efetivamente.

\subsection{GERENCIAMENTO DE CRISES}

Nas últimas décadas foram observadas uma série de crises das mais diferentes dimensões (Figura 1) que atingiram desde o cidadão comum até governos, onde muitas delas, infelizmente, deixaram grandes cicatrizes no tempo. Essas crises tem trazido a tona a vulnerabilidade em que se encontram a população, organizações e governos no que tange o enfreitamento de crises.

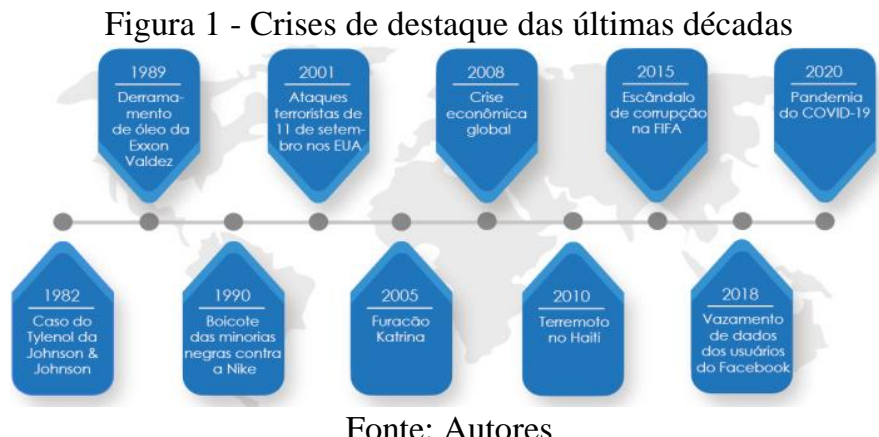

Esta série de crises e acidentes atraiu o interesse de pesquisadores de várias disciplinas, resultando no surgimento de uma literatura rica, no entanto fragmentada, sobre crise. Segundo Cania e Korsita (2015) o desenvolvimento de várias crises tornou-se parte integrante dos negócios modernos. A palavra "crise", de origem antiga e clássica, em grego significa "julgamento", ou seja, trata-se de um momento para tomada de decisão.

Atualmente, o termo crise é usado para descrever eventos de baixa probabilidade e grandes consequências que afetam as estruturas, valores ou padrões de um sistema social, podendo ameaçar severamente a integridade de uma organização (Wang \& Belardo, 2009), nos 
quais, em uma situação altamente pressurizada e incerta, decisões cruciais devem ser tomadas (Rosenthal, Charles, \& Hart, 1989).

Crises também podem ser definidas como fenômenos em evolução que lidam com muita informação (Ramète, Lamothe, Lauras, \& Benaben, 2012) e intensivos em conhecimento (Coombs, 1999). Lauras et al. (2015) classificam as crises por natureza (técnicas, humanas, políticas, legais, éticas, econômicas ou desastres naturais), evento de perigo, velocidade de ação ou por intensidade. Para Ramète et al. (2012) uma crise é composta por três dimensões: fatores de complexidade (tipo de crise, responsabilidade humana), fatores de gravidade (medição de danos) e efeitos (impactos). Sendo assim, uma crise pode evoluir e variar suas dimensões.

O Institute for Crisis Management (2020) acompanhou um total de 760.099 notícias negativas no mundo em 2019 e identificou que as organizações continuam lutando para se recuperar de uma variedade de problemas, tanto não intencional e quanto auto-infligido. A origem das principais crises identificadas no relatório é apresentada na Figura 2 conforme incidência dos casos.

Figura 2 - Origem das principais crises em 2019

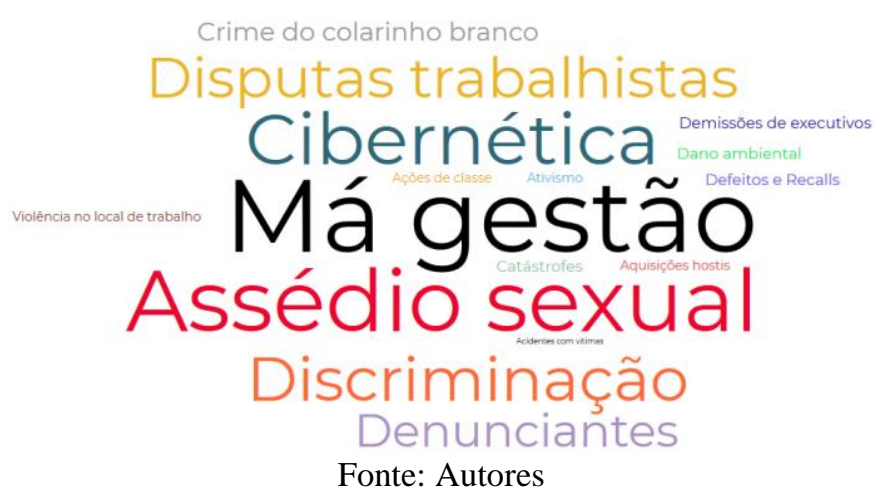

Antes de haver uma crise, tem-se o risco. Trata-se de um evento incerto que caso aconteça pode gerar impactos positivos ou negativos. Já um gatilho é uma espécie de limiar de transição entre o risco e a sua materialização, sendo o risco com impacto negativo, esse gatilho é representado por situações de perigo e emergência que se tornam crises se a resiliência do sistema e a preparação forem insuficientes para responder a estes eventos (Wybo, 2004). A crise pode ainda gerar novos riscos, que podem ocasionar novas crises. $\mathrm{O}$ gerenciamento de riscos inclui processos de planejamento, identificação, análise qualiquantitativa, planejamento das respostas, implementação das respostas e monitoramento dos riscos (PMI, 2017).

Há muitos modelos que permitem uma compreensão do ciclo de vida do gerenciamento de crises, Mitroff (1988) distingue cinco etapas: detecção de sinal; preparação/prevenção; contenção; recuperação; e aprendizagem. Para Hernantes et al. (2013), essa proposta pode ser resumida, considerando que detecção de sinal, preparação e prevenção estão relacionadas ao 
estágio pré-evento, contenção/resposta ao evento e recuperação e aprendizagem ao pós-evento. Essa simplificação vai ao encontro da proposta de Coombs (1999) que considera o gerenciamento de crises composto por três fases: pré-crise, durante a crise e pós-crise.

Segundo Ponis e Koronis (2012), na fase pré-crise, a gerência deve tentar prever as crises, a probabilidade de ocorrência e se preparar o máximo possível. Durante a crise, as coisas mudam drasticamente, pois o tempo para a tomada de decisões é reduzido e há condições de alta incerteza. Na última fase, a administração deve procurar restaurar sua reputação e realizar mudanças em sua estrutura, políticas, cultura e mecanismos de controle, na tentativa de se recuperar da crise e aprimorar o aprendizado individual e organizacional (Anttila (2014).

Por mais que uma crise possa abalar um sistema, ela pode por fim a longos períodos de aprisionamento institucional e possibilitar grandes mudanças organizacionais. $\mathrm{Na}$ literatura, essas situações são conhecidas como conjunturas críticas ou janelas de oportunidade (Capoccia \& Kelemen, 2007). Na teoria, uma crise pode funcionar como um catalisador para a aprendizagem (Broekema, Porth, Steen, \& Torenvlied, 2019).

\subsection{PRÁTICAS E DESAFIOS DA GC EM TEMPOS DE CRISE}

As crises organizacionais são consideradas eventos que produzem desafios adicionais para a GC, principalmente porque o conhecimento complexo, polimórfico, estruturado e não estruturado deve ser eficientemente aproveitado, processado e disseminado para os atores internos e externos adequados, sob restrições específicas de tempo, mídia e ambiente (Ponis \& Koronis, 2012). Uma organização precisa empregar diferentes estratégias de conhecimento em diferentes fases de uma crise para atender às diferentes necessidades de conhecimento e alcançar os resultados desejados no gerenciamento de crise (Wang \& Belardo, 2009).

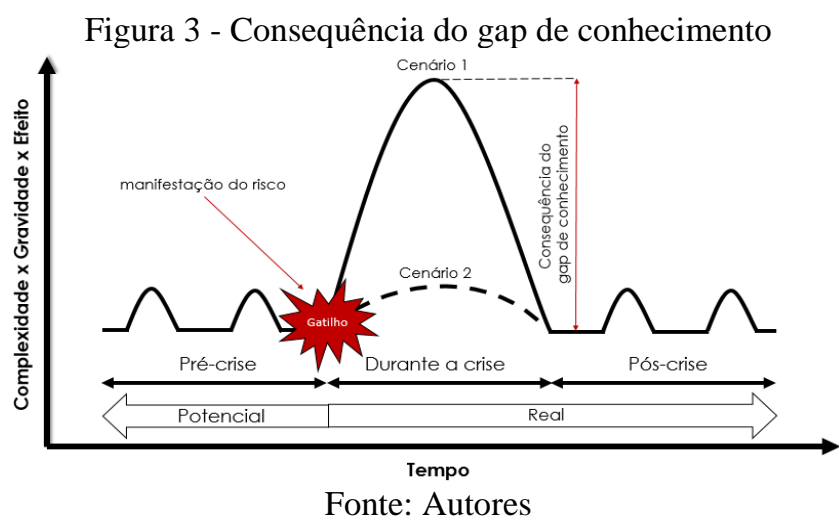

A Figura 3 caracteriza uma situação hipotética onde no Cenário 1 a organização não se preparou adotando estratégias de conhecimento adequadas e tampouco implementou ações de 
modo eficaz vivenciando uma grave crise. Por outro lado, no Cenário 2 se tem uma organização que se preveniu adotando estratégias para utilizar todo o conhecimento disponível e adquirido e implementou medidas agilmente que mitigaram a crise até sua devida extinção.

Crises organizacionais obrigam as empresas a aprender e tomar decisões rapidamente, processando os recursos de conhecimento necessários (Ponis \& Koronis, 2012). O aprendizado é de importância central devido às conseqüências físicas, econômicas, ecológicas e sociais devastadoras e duradouras que as crises podem ter (Broekema et al., 2019). Ao realizar esses processos de aprendizagem, as organizações podem desenvolver estratégias, proativas e reativas, úteis para lidar com as crises nos negócios antes que o próximo evento ocorra (Wang \& Belardo, 2009). As situações de crise testam a preparação de organizações, sistemas, processos, das pessoas e principalmente a resiliência e liderança (Germain, 2010).

Paraskevas et al. (2013) acrescentam que a liderança em crises desempenha um papel importante na implementação de estratégias de gerenciamento de conhecimento de codificação e personalização por meio de seu apoio explícito aos planos de gerenciamento de crises e da exploração de suas habilidades de liderança visionárias e proativas. Broekema et al. (2019) acrescentam que a liderança desempenha um papel central no gerenciamento de crises.

Antes que uma crise exploda, é necessário adquirir e armazenar conhecimento. Isso pode ser obtido de fontes internas e externas (informações históricas sobre sintomas, preparação, custos de intervenção) (Kyobe, 2010). O conhecimento deve ser armazenado em repositório centralizado de conhecimento servindo para: manutenção de informações históricas; recurso para codificar e criar novos conhecimentos; organização; melhores práticas e estratégias comprovadas de gerenciamento de crises; recomendações para cursos de ação antes, durante e após uma situação de crise (Racherla \& Hu, 2009), bem como incluir "páginas amarelas" com informações sobre quem é especialista em quê e como contatar (Paraskevas et al., 2013).

Tecnologias da informação facilitam o desenvolvimento de intranets e extranets da empresa, bem como o design de sites de crise especializados e centros de aprendizado on-line (Paraskevas et al., 2013). Sistemas de Suporte à Decisão da Informação também podem ser implantados segundo Ramète et al. (2012). Hernantes et al. (2013) apontam que modelos de simulação também permitem que especialistas compreendam as consequências de determinadas políticas a curto e longo prazos, melhorando o conhecimento para situações futuras de crise.

Uma revisão da literatura sobre gerenciamento de crises indica que a maioria das crises resulta da falta de gerenciamento e compartilhamento adequados do conhecimento (Kyobe, 2010). Ponis e Koronis (2012) afirmam que é essencial que os atores envolvidos em uma crise possuam o conhecimento do que aconteceu, os regulamentos relevantes e as visões da equipe 
antes de se envolverem na comunicação da crise às partes interessadas (stakeholders), à mídia e ao público em geral. Kaewkitipong, Chen e Ractham (2016) informam que as mídias sociais são cada vez mais aceitas como ferramentas eficazes de compartilhamento de conhecimento em crises, e diferentes aplicações foram usadas no combate às crises recentes em todo o mundo.

Racherla e $\mathrm{Hu}$ (2009) sugerem que os sistemas de gerenciamento de conhecimento colaborativo podem melhorar a preparação e o gerenciamento de crises. A IBM, por exemplo, após terremoto em 2011 no Japão criou, à nível mundial, uma rede de conhecimento interno para fomentar a colaboração e agilizar o compartilhamento de conhecimento na empresa (Muller \& Chua, 2012). O desenvolvimento de recursos humanos pode atuar como um facilitador na melhoria de redes e colaboração para lidar com crises (Germain, 2010).

Os atores envolvidos nas crises necessitam de treinamento e revisar constantemente os processos de crise, Germain (2010) afirma que as simulações de crise em treinamentos desempenham um papel importante na preparação dos funcionários para situações de emergência e crise e sugere enfase no treinamento prático, pois pode ser mais valioso durante a crise do que o e-learning. Paraskevas et al. (2013) ressaltam que todo o material de treinamento deve ser armazenado nos repositórios para reutilização em outras crises.

\section{METODOLOGIA}

A natureza exploratória qualitativa desta pesquisa consiste em uma revisão sistemática de literatura por intermédio da coleta e análise de diversas publicações sobre o campo comum entre as temáticas de GC e gerenciamento de crises. O estudo teve foco nas publicações que foram realizadas nesta área sobre estudos teóricos e empíricos das últimas quatro décadas, analisando diferentes abordagens sobre a temática nos mais diversos setores da economia. Essas publicações foram divulgadas em diferentes periódicos, conferências e através de livros.

O trabalho utilizou para a pesquisa das publicações a base Scopus ${ }^{\circledR}$, a maior base de dados de resumos e citações de literatura científica revisada por pares no mundo. Esta base possui natureza multidisciplinar e abrange as áreas de ciência, tecnologia, medicina, ciências sociais, artes e humanidades, ou seja, consiste em uma base de dados aderente ao objeto de estudo, visto que vai ao encontro da multidisciplinaridade da GC e do gerenciamento de crises.

Neste estudo foi empregada a abordagem proposta por Coombs (1999) para o gerenciamento de crises que considera a crise composta por três fases: pré-crise, durante a crise e a pós-crise. Esta definição levou em conta que os modelos apresentados por outros autores podem ser simplificados para esta concepção, sem apresentar qualquer lacuna, uma vez que 
contém todas as demais fases dispostas neste modelo trifásico.

Foram determinadas duas estratégias para a GC, a codificação e a personalização, conforme Hansen, Nohria e Tierney (1999). Essas estratégias podem ser relacionadas a diferentes formas de se disseminar o conhecimento. A codificação implica codificar o conhecimento e armazená-lo em bases de dados que podem ser consultadas e utilizadas por qualquer profissional. Já na personalização, o conhecimento está fortemente ligado à pessoa que o desenvolveu e é compartilhado principalmente por meio de contatos de pessoa a pessoa.

A partir destas definições, a literatura relevante foi revisada rigorosamente para construir um conjunto teórico de elementos, ações e artefatos dos campos da GC, gerenciamento de riscos, gerenciamento de crises, que resultou em um framework teórico prescritivo de processos fornecendo orientações gerais sobre os procedimentos da GC necessários a prevenção, combate e recuperação de crises.

\section{RESULTADOS E ANÁLISES}

\subsection{FASE 1: PRÉ-CRISE}

Antes de mais nada é necessário definir o(a) responsável pela temática de crises na organização, pois ele(a) será o ponto focal na coordenação das atividades relacionadas ao gerenciamento de crises na empresa. Espera-se que tenha habilidades interpessoais. Deve-se então institucionalizar a área ou setor, definindo diretrizes, responsabilidades e formalização junto a organização. O próximo passo é planejar o gerenciamento dos riscos organizacionais a fim de estabelecer diretrizes de como as atividades serão gerenciadas e executadas.

Sugere-se mapear os detentores do conhecimento na organização, especialistas em cada área, para serem acionados quando necessário como destacado por Kyobe (2010). Uma próxima etapa é a identificação e categorização dos riscos (técnicos, de gerenciamento, comercial, externo), atividades que podem ser realizadas através de consulta com especialistas, entrevistas, diagrama de causa e efeito (Ishikawa) ou técnica de brainstorming (PMI, 2017), resultando em uma estrutura analítica de riscos. Este é um documento deve ser atualizado regularmente.

Identificado os riscos, o próximo passo é analisar qualitativamente (probabilidade e impacto) a fim de priorizar os riscos. Após esta etapa é necessário analisar quantitativamente os riscos, onde é avaliado o aspecto financeiro do efeito combinado pelos riscos. Estas atividades fornecem subsídios para a etapa de planejar respostas aos riscos a fim de desenvolver alternativas, selecionar estratégias e definir ações para lidar com a exposição geral aos riscos e 
também o devido tratamento. Neste plano deve conter os gatilhos associados a cada risco, que servirão de sinal que um risco está prestes a se materializar e que ações precisarão ser tomadas.

Uma outra etapa importante é desenvolver o plano de comunicação da organização, definindo o quê, quando, como e para quem devem ser trasmitidas as informações quando eventos ocorrerem. Kaewkitipong et al. (2016) recomendam dividir o planejamento da comunicação de crises em fases, cada uma enfatizando atividades diferentes. A comunicação com os stakeholders é essencial para diminuir a ansiedade e obter cooperação (Hernantes et al., 2013). Os riscos precisam ser monitorados continuamente e devem estar no radar do gestor, pois assim que houver um gatilho, as respostas aos riscos devem ser implementadas.

Após a implementação das respostas, caso o risco tenha sido evitado, as lições aprendidas devem ser devidamente coletadas e armazenadas em um repositório de conhecimento conforme ensinado por Racherla e Hu (2009) e Paraskevas et al. (2013). Havendo materialização do risco, os stakeholders devem ser imediatamente comunicados e iniciada a preparação do plano de contingência, uma vez que outros impactos podem ter sidos gerados ou a dimensão dos efeitos diferente da prevista. Cessando os impactos, as lições aprendidas devem ser coletadas e armazenadas. Caso os efeitos permaneçam, mas sem comprometer a integridade organizacional (física, humana, financeira, imagem, por exemplo) um novo plano de contigência deverá ser preparado e implementado.

\subsection{FASE 2: DURANTE A CRISE}

No entanto, caso os efeitos permaneçam e comprometam a integridade organizacional ou um evento de alto impacto ocasionado por um risco não identificado ou de natureza desconhecida venha acontecer, torna-se necessário a constituição de um comitê de crises. Este comitê poderá ser composto por especialistas multidisciplinares das áreas de origem do evento e impactadas por ele. Além do conhecimento técnico, o comitê deverá apresentar habilidades de negociação, comunicação e principalmente liderança, pois esta desempenha um papel central no gerenciamento de crises (Paraskevas et al., 2013; Anttila, 2014; e Broekema et al., 2019).

O comitê, com base no repositório de conhecimento organizacional deverá realizar um diagnóstico da crise, sob as diferentes perspectivas, para que se tenha um entendimento sistêmico do cenário. Após esta compreensão, e não antes, os stakeholders deverão ser comunicados, conforme previsto no plano de comunicação. Ele deve manter uma periodicidade em atualizar os stakeholders rapidamente quanto ao status da crise e evitar a disseminação de informações falsas. As mídias sociais podem ser utilizadas como ferramentas para o 
compartilhamento de conhecimento no combate às crises conforme Kaewkitipong et al. (2016).

O comitê de crise deverá verificar se a organização detém o conhecimento para tratar a crise, caso positivo, deverá planejar as ações necessárias para lidar com a crise. No entanto, caso a organização não possua o conhecimento necessário para tratar a crise ela pode optar por adquirir o conhecimento de terceiros ou treinar seus profissionais, desde que haja tempo e recursos disponíveis. Cania e Korsita (2015) destacam que para ter sucesso com crises intensas, a gerência organizacional deve desenvolver e estabelecer novas habilidades.

Tanto o conhecimento adquirido de terceiros quanto o desenvolvido internamente devem ser armazenados. De posse do conhecimento, o comitê deverá planejar as ações para lidar com a crise e implementar as medidas. A técnica de brainstorm para resposta a crise é uma opção, conforme bem empregado pela IBM $^{\circledR}$ no terremoto e tsunami no Japão em 2011 (Muller \& Chua, 2012). Caso sejam detectados novos riscos, estes precisam ser identificados, analisados, planejadas suas respostas e monitorados. As ações implementadas devem ser monitoradas até que a integridade organizacional não esteja mais ameaçada. No entanto, as ações sendo insuficientes e a integridade continue ameaçada, novas ações deverão ser buscadas.

\subsection{FASE 3: PÓS-CRISE}

Este é o momento de recuperação e aprendizagem da organização. Nesta fase o comitê deve preparar o plano de recuperação e implementar estas medidas afim de retornar a organização mais próxima do estado anterior a crise, uma situação estável, ou condições melhores. É preciso realizar uma avaliação geral da crise para verificar quão resiliente esta foi (Ramète et al., 2012) e solicitar feedback dos stakeholders. Esse aprendizado deve ser disseminado na organização podendo ser utilizada técnica de storytelling que vem sendo empregada neste cenário (Kopp, Nikolovska, Desiderio, \& Guterman, 2011).

Estas lições devem ser armazenadas para se tornarem conhecimento organizacional. Esta fase é uma oportunidade para a organização reavaliar as competências dos seus profissionais, verificar as falhas de processos e a necessidade de tecnologias. Através da aprendizagem induzida por crises, uma organização pode melhorar suas atividades de resposta e incorporar medidas para evitar crises futuras (Broekema et al., 2019).

A Figura 4 sintetiza todos os processos apresentados, resultando em um framework que incorpora princípios de GC para aprimorar a eficácia do planejamento e gerenciamento de crises e assim apoiar organizações nestes tempos difíceis. 
Figura 4 - Framework de gestão do conhecimento em tempos de crise

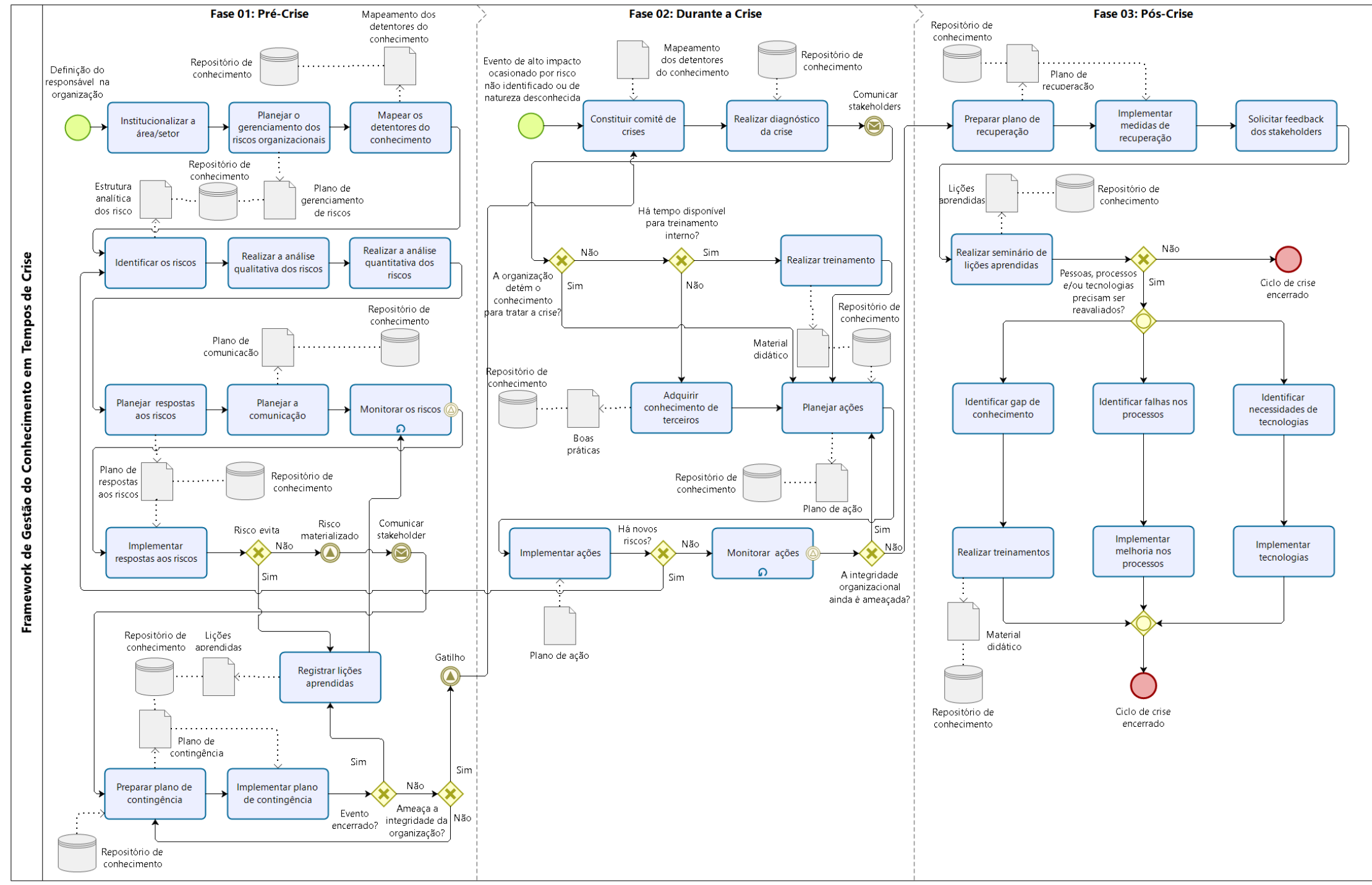

Fonte: Autores

\section{5 S ciudad 8 EGC TECNOPUC}




\section{CONSIDERAÇÕES FINAIS}

Gerenciar situações complexas como crises é uma tarefa desafiadora para as organizações, principalmente devido à heterogeneidade dos parceiros envolvidos e à natureza crítica de tais eventos (Lauras et al., 2015). A gestão do conhecimento tem sido reconhecida como uma importante disciplina capaz de apoiar a gestão da complexidade em tempos de crise, tornando-se essencial nestes períodos incertos (Coombs, 1999; Wang \& Belardo, 2009; Kyobe, 2010; Blackman et al., 2011; Ponis \& Koronis, 2012; Cania \& Korsita, 2015; Lauras et al., 2015). O presente estudo contribui neste aspecto, trazendo uma estrutura organizada e orientada pela gestão do conhecimento afim de apoiar o gerenciamento de crises.

Em razão de não se restringir a um tipo específico de crise, mas apresentar estratégia genérica conforme alguns autores citados por Racherla e Hu (2009), o framework resultante do estudo poderá ser utilizado como material de referência para consulta pelas mais diversas organizações, desde microempresas à multinacionais, do setor de turismo à manufatura, de caráter público ao privado. Vale destacar que este é um modelo teórico e cabe sua aplicação em um caso real ou simulado, bem como a aceitação da academia para a sua devida validação.

Uma vez que ainda há pouca pesquisa que vincule explicitamente gestão do conhecimento e gerenciamento de crises (Wang \& Belardo, 2009; Ponis \& Koronis, 2012; Paraskevas et al., 2013), a proposta apresentada contribui para fortalecer este link entre conhecimento e crise, bem como trazer novos insights para estudiosos da temática criarem novos modelos, ferramentas e técnicas utilizando o conhecimento como uma arma letal para a prevenção e combate a crises organizacionais.

Destacam-se como possíveis desdobramentos desta pesquisa a aplicação do framework em um exemplo hipotético; a simulação de um evento real e comparação e análise das estratégias adotadas e resultados obtidos; e o desenvolvimento de uma plataforma colaborativa que represente o framework contendo todos os processos automatizados, objetos e artefatos para a fácil e ágil criação, armazenamento, compartilhamento e uso do conhecimento de crise.

\section{AGRADECIMENTOS}

Este artigo foi apoiado pelo Departamento de Engenharia e Gestão do Conhecimento (EGC) da Universidade Federal de Santa Catarina (UFSC) e financiado pela Coordenação de Aperfeiçoamento de Pessoal de Nível Superior (CAPES), fundação vinculada ao Ministério da Educação do Brasil. 


\section{REFERÊNCIAS}

Anttila, U. M. A. (2014). Human security and learning in crisis management. Journal of Humanitarian Logistics and Supply Chain Management, 4(1), 82-94.

Argote, L., McEvily, B., \& Reagans, R. (2003). Managing knowledge in organizations: An integrative framework and review of emerging themes. Manag. Science, 49(4), 571-582.

Bhatt, G. D. (2001). Knowledge management in organizations: examining the interaction between technologies, techniques, and people. Journal of Knowledge Management, $5(1), 68-75$.

Blackman, D., Kennedy, M., \& Ritchie, B. (2011). Knowledge management: The missing link in DMO crisis management? Current Issues in Tourism, 14(4), 337-354.

Broekema, W., Porth, J., Steen, T., \& Torenvlied, R. (2019). Public leaders' organizational learning orientations in the wake of a crisis and the role of public service motivation. Safety Science, 113, 200-209.

Cania, L., \& Korsita, B. (2015). Knowledge management: The key to sustainability of economic crisis. Mediterranean Journal of Social Sciences, 6(1), 548-553.

Capoccia, G., \& Kelemen, R. D. (2007). The study of critical junctures. Theory, narrative, and counterfactuals in historical institutionalism. World Politics, 59(3), 341-369.

Coombs, T. (1999). On-going crisis communication: planning, managing and responding. London: Sage Publications.

Davenport, T., \& Prusak, L. (1998). Working knowledge: managing what your organisation knows. Boston: Harvard Business School Press.

Evans, C., (2003). Managing for knowledge: HR's strategic role. Oxford: BH.

Hansen, M. T., Nohria, N., \& Tierney, T. (1999). What's your strategy for managing knowledge? Harvard Business Review, 77(2), 106-116.

Hernantes, J., Rich, E., Laugé, A., Labaka, L., \& Sarriegi, J. M. (2013). Learning before the storm: modeling multiple stakeholder activities in support of crisis management, a practical case. Technological Forecasting and Social Change, 80(9), 1742-1755.

Institute for Crisis Management. (2020). ICM annual crisis report. South Bend: ICM.

International Organization for Standardization. (2018). ISO 30.401: 2018 Knowledge management systems: requirements. Geneva: ISO.

Kaewkitipong, L., Chen, C. C., Ractham, P. (2016). A community-based approach to sharing knowledge before, during, and after crisis events: A case study from Thailand. Computers in Human Behavior, 54, 653-666.

Kopp, D. M., Nikolovska, I., Desiderio, K. P., Guterman, J. T. (2011). "Relaaax, I remember the recession in the early 1980s...": Organizational storytelling as a crisis management tool. Human Resource Development Quarterly, 22(3), 373-385.

Kyobe, M. (2010). A knowledge management approach to resolving the crises in the information systems discipline. Journal of Systems and Information Technology, 12(2), 161-173.

Lauras, M., Truptil, S., \& Bénaben, F. (2015). Towards a better management of complex emergencies through crisis management meta-modelling. Disasters, 39(4), 687-714. 
Mitroff, I. I. (1988). Crisis management: cutting through the confusion. Sloan Management Review (Winter), 29(2), 15-20.

Muller, M., \& Chua, S. (2012). Brainstorming for Japan: rapid distributed global collaboration for disaster response. Proceedings of the SIGCHI Conference on Human Factors in Computing Systems. 2727-2730.

Paraskevas, A., Altinay, L., McLean, J., \& Cooper, C. (2013). Crisis knowledge in tourism: types, flows and governance. Annals of Tourism Research, 41, 130-152.

Project Management Institute - PMI. (2017). Guia PMBOK (6a ed). Filadélfia: PMI.

Polanyi, M. (1966). The tacit dimension. London: Routledge.

Ponis, S. T., \& Koronis, E. (2012). A knowledge management process-based approach to support corporate crisis management. Knowledge and Process Management, 19(3), 148159.

Racherla, P., \& Hu, C. (2009). A framework for knowledge-based crisis management in the hospitality and tourism industry. Cornell Hospitality Quarterly, 50(4), 561-577.

Ramète, G. M., Lamothe, J., Lauras, M., \& Benaben, F. (2012). A road crisis management metamodel for an information decision support system. In: 6th IEEE International Conference on Digital Ecosystems and Technologies.

Rosenthal, U., Charles, M. T., \& Hart, P. (1989). Coping with crisis: the management of disasters, riots and terrorism. Springfeld: Charles C. Thomas.

Wang, W. -T., \& Belardo, S. (2009). The role of knowledge management in achieving effective crisis management: a case study. Journal of Information Science, 35(6), 635-659.

Wybo, J. L. (2004). Mastering risks of damage and risks of crisis: the role of organisational learning. International Journal of Emergency Management, 2, 22-34.

Zack, M. H. (1999). Developing a knowledge strategy. California Management Review, 41(3), 125-145. 